\title{
Nutrient-induced hyperosmosis evokes vasorelaxation via TRPV1-mediated endothelium-dependent hyperpolarization in normal and colitis mice
}

\author{
Yanjun Guo ${ }^{1}$, Cheng Luํㄹ Luyun Zhang ${ }^{1}$, Hanxing Wan ${ }^{1}$, Enlai Jiang ${ }^{1}$, Yao Chen ${ }^{1}$, and \\ Hui Dong ${ }^{2}$ \\ ${ }^{1}$ Xinqiao Hospital, Army Medical University \\ ${ }^{2}$ University of California, San Diego
}

May 14, 2020

\begin{abstract}
Background and Purpose: Although human blood flows are redistributed into the mesenteric circulation after meals, it is not well understood how postprandial nutrients induces vasorelaxation of mesenteric micro-arterioles and whether this process is involved in the pathogenesis of colitis. Experimental Approach: We used an auto dual wire myograph system, fluorescence imaging system and DSS-induced colitis mouse model to investigate the roles and mechanisms of nutrient-induced mesenteric relaxation in health and disease. Key Results: We found that acute application of glucose and sodium induced endotheliumdependent relaxation of human and mouse mesenteric micro-arterioles via a hyperosmotic action, which also stimulated Ca2+ influx through endothelial TRPV1 channels. The nutrient-induced vasorelaxation was almost abolished by selective blockers for TRPV1, IKCa and SKCa channels, but marginally altered by inhibition of nitric oxide production. The nutrient-induced hyperosmosis also activated functional activities of $\mathrm{Na}+/ \mathrm{K}+$-ATPase and $\mathrm{Na}+/ \mathrm{Ca} 2+$-exchanger to further reduce [Ca2+]i in vascular smooth muscle cells. Moreover, hyperosmosis-induced endothelium-dependent hyperpolarization was significantly impaired in colitis mouse model. Conclusion and Implications: Our study provides the first evidence that nutrient-induced hyperosmosis stimulates endothelial TRPV1/Ca2+/EDH signaling pathway to eventually evoke vasorelaxation of mesenteric micro-arterioles, which may contribute to the pathogenesis of colitis as well.
\end{abstract}

\section{Hosted file}

Main Manuscript.doc available at https://authorea.com/users/321786/articles/450971-nutrientinduced-hyperosmosis-evokes-vasorelaxation-via-trpv1-mediated-endothelium-dependenthyperpolarization-in-normal-and-colitis-mice

\section{Hosted file}

Figures.doc available at https://authorea.com/users/321786/articles/450971-nutrientinduced-hyperosmosis-evokes-vasorelaxation-via-trpv1-mediated-endothelium-dependenthyperpolarization-in-normal-and-colitis-mice 